\title{
EXTRACTION AND REFINEMENT OF FINGERPRINT ORIENTATION FIELD
}

\author{
Pierluigi Maponi, Riccardo Piergalliniand Filippo Santarelli \\ Department of Mathematics, University of Camerino, Camerino, Italy
}

\begin{abstract}
We propose a gradient-based method to extract the orientation field of a fingerprint image, and an iterative algorithm to refine and regularise this field. The formulation of this iterative algorithm is based on two new integral operators, which are described together with their main properties. A preprocessing step is also proposed in order to enhance the performance of the whole procedure. The results of our tests on real fingerprint images are provided to show the performance of the proposed approach.
\end{abstract}

\section{KEYWORDS}

Fingerprint analysis, Preprocessing, Equalisation, Segmentation, Orientation Extraction, Orientation Refinement

\section{INTRODUCTION}

Biometrics is a widely studied field, engaging scientists in several disciplines such as engineering, biology, mathematics and law. Interest in biometric authentication or recognition has grown in the last decades for medical, forensic, government and socio-economic applications. Fingerprint is one of the most distinctive biometric trait, different even for twins, with low storage needs and low cost acquisition systems; hence it is one of the most used biometric measure. Recent advances in computer science and improvements of hardware performances allowed the development of several automated fingerprint recognition systems. Such systems operate in two different modes: verification and identification. Verification in mostly used for civilian purposes, such as restricted resources access control, where an input fingerprint is compared with a database of already enrolled fingerprints; in the event that the input is present in the database, the verification is successful and the access granted. Identification is intended to find the identity ofa person, and a fingerprint is compared with a database, that may not contain it; this is mainly used by law enforcement agencies for investigation purposes and the analysis of the crime scene.

Fingerprint images are characterised by a very particular structure formed by several almost parallel curves, which are usually called ridges. A typical recognition system consists of the following steps:

1. Image acquisition, through inked paper scanning or directly by newer fingerprint scanners.

2. Preprocessing, aimed to noise removal and contrast enhancement.

3. Feature extraction, where some selected features are computed from the fingerprint for later use in the matching stage, or possibly stored in a database. In this stage is often present a codification procedure to reduce storage needs and to boost matching speed.

4. Postprocessing, intended to improve the extracted features or to remove bad ones.

Natarajan Meghanathan et al. (Eds) : ICCSEA, WiMoA, SPPR, GridCom, CSIA - 2017

pp. 57-68, 2017. (C) CS \& IT-CSCP 2017

DOI : $10.5121 /$ csit.2017.71105 
5. Matching, where a comparison is performed between the extracted features and the ones stored in the database [1-3].

The orientation field estimation, that is the extraction of information about ridge flow, is frequently present inside a fingerprint processing algorithm. Indeed the orientation field is used for classification [4], to detect singular points [5], to detect fingerprints alterations [6],for registration before matching [7], to improve matching performance $[8,9]$ and as a matching feature in itself [10]. Thus, the computation of a reliable orientation field from a fingerprint image is a problem of great interest and many techniques have been already developed. Usual approaches to this problem are: gradient-based, slit- and projection-based techniques, frequency domain orientation estimation. Poor quality fingerprints may contain creases, scratches, discontinuous ridge patterns and no-signal areas, which yield noise in the orientation fields, regardless of the chosen approach [3]. Hence, several methods have been proposed for postprocessing in order to reduce the orientation field unreliability, such as the orientation regularisation by using coherence criteria [11], neural network classification of unreliable orientations [12], multi-scale analysis for the correction of elements that change among different scales [13]. Other interesting contributions come from [14], where the correction of the estimated orientation field is based on the information coming from a global orientation model, and [15], where a probabilistic approach to the orientation field regularisation is introduced. [16] and [3] provide a detailed description of other interesting approach for the orientation field enhancement.

Throughout this paper we focus on the second and third stages of the typical fingerprint recognition system. A new algorithm for the estimation of the orientation field and its refinement is proposed. The estimation of the orientation field is performed by a gradient based method: a group of directional gradient masks are chosen and convolved with the image, and their responses are combined to get an orientation field. The enhancement step is based on two operators that can be applied to the orientation field to detect singular points, to smooth the integral curves around singular points, and to iteratively remove noise.

In Section 2 a detailed description of the proposed method is provided. In Section 3 we describe some numerical results obtained with the proposed method. In Section 4 we provide some conclusions.

\section{ALGORITHM}

Let $I$ be an $N \times M$ grey scale image, where each element $I(i, j)$ with $i=1, \ldots, N$ and $j=1, \ldots, M$ is a grey level ranging from 0 to 255 .

Fingerprint images require the definition of the direction and orientation fields; here we use the same distinction introduced by Sherlock and Monro [17]. Given a complex numberz $\in \mathbb{C}$, its phase is the angle $\theta \in[0,2 \pi)$ that it forms with the positive real axis. Every complex number $z$ having the same phase angle $\theta$ defines the same direction, regardless its modulus. The set of all the possible directions can be naturally identified with the unit circle $S^{1}$. Consider the straight line $\gamma$ given by $t z$, with $t \in \mathbb{R}$; it forms an angle $\phi$ with the positive real axis that lies in the range $[0, \pi)$, since $\gamma$ is invariant by rotation through integer multiples of $\pi$. Every complex number $z$ having the same angle $\phi$ defines an orientation, regardless its modulus. The set of all the possible orientations can be naturally identified with the projective circle $P^{1}$. Note that the concept of vector field, that is a mapping from the image to $S^{1}$, is unsuitable to describe the ridge flow of a fingerprint; hence our aim is to compute an orientation field, that is a map from the image to $P^{1}$.

The proposed algorithm is composed by the two steps: preprocessing, described in Section 2.1, and orientation extraction, outlined in Section 2.2. 


\subsection{Preprocessing}

The preprocessing stage combines the image equalisation, the segmentation and the ridges amplification, and is an important preliminary step that yields reliability to the orientation estimation.

The very first operation to be performed is the image scaling to the full grey level range, i.e. $1, \ldots, 255$, applying a linear transformation. The grey level histogram is then computed and smoothed convolving it with a Gaussian kernel.

Fingerprint images coming from real test cases often produce an unbalanced histogram, implying an image either too bright or too dark. So a more balanced distribution of grey levels can be obtained through an iterative process, where an adaptive threshold grey level $\bar{x}$ on the histogram is computed in such a way that $\bar{x}$ is the mean value between the right and the left means; let us call $m_{\mathrm{L}}$ and $m_{\mathrm{R}}$ the final left and right means respectively.

Since small grey values variations around 0 or 255 do not provide very useful information, we can remove them. Given two real numbers $t_{\mathrm{L}}, t_{\mathrm{R}} \in[0,1]$ we compute two threshold values $x_{\mathrm{L}}$ and $x_{\mathrm{R}}$ on the histogram so that $x_{\mathrm{L}}=t_{\mathrm{L}} m_{\mathrm{L}}$ and $x_{\mathrm{R}}=255-t_{\mathrm{R}}\left(255-m_{\mathrm{R}}\right)$; we define the following continuous function:

$$
f(x)=\left\{\begin{array}{cl}
0 & x \leq x_{\mathrm{L}} \\
\frac{x-x_{\mathrm{L}}}{\bar{x}-x_{\mathrm{L}}} \cdot 127 & x_{\mathrm{L}}<x \leq \bar{x} \\
127+\frac{x-\bar{x}}{x_{\mathrm{R}}-\bar{x}} \cdot 128 & \bar{x}<x \leq x_{\mathrm{R}} \\
255 & x>x_{\mathrm{R}}
\end{array}\right.
$$

that maps grey levels less than $x_{\mathrm{L}}$ to $0, \bar{x}$ to 127 , and the ones greater than $x_{\mathrm{R}}$ to 255 . Finally we can obtain the equalised image $I_{\mathrm{E}}$ by computing

$$
I_{\mathrm{E}}(i, j)=f(I(i, j)), i=1, \ldots, N, j=1, \ldots, M .
$$

The preliminary step to compute a significant mask is to remove marginal lines with very small variations in grey values. A minimum-allowed variation threshold $\tau_{\mathrm{V}}$ is set; from the image borders to the centre, for each row or column its maximum variation $V$ is computed and if $V<\tau_{\mathrm{V}}$ the row or column is removed.

Acquisitions coming from fingerprint cards, because of camera misalignment, often present oblique lines nearby the border that must be excluded from further analysis. So, we apply to the image a two-dimensional $5 \times\left[\frac{M}{4}+1\right]$ filter, where [·] is the rounding operator, with the kernel of the form:

$$
(1,1,0,-1,-1)^{\mathrm{T}} \cdot(1,1, \ldots, 1,1),
$$

and $\mathrm{T}$ is the matrix transposition operator. This filter's response has a high absolute value where there are strong vertical changes in grey values. The outputs of this filter and of the transposed filter are combined together with a line-fitting algorithm to detect oblique lines; the image part outside those lines is removed from further steps.

Fingerprints may contain handwritten text and other artifacts, that are removed in this step. We perform a simple image binarisation and morphology operations to exclude small or thin connected components. The image gradient is also binarised, dilated, and worked with classical morphology techniques, so that we obtain a second mask stricter than the previous. In this way artifacts can be removed and foreground be segmented.

In the following we proceed to amplify the ridges. Let $I_{\mathrm{R}}$ be the restriction of the initial image $I$ to the significant mask obtained in the segmentation step. A matrix $I_{M}$ is computed by taking the 
maximum in a circular neighbourhood of every pixel of $I_{\mathrm{R}}$; let $I_{\mathrm{m}}$ be the matrix computed analogously by considering the circular minimum filter applied to $I_{\mathrm{R}}$. In order to emphasise the alternation of ridges and valleys, two threshold values $t_{\mathrm{M}_{1}}$ and $t_{\mathrm{M}_{2}}$ are chosen for $I_{\mathrm{M}}$ and another two values $t_{\mathrm{m}_{1}}$ and $t_{\mathrm{m}_{2}}$ for $I_{\mathrm{m}}$; the values of $I_{\mathrm{M}}$ in the range $\left[t_{\mathrm{M}_{1}}, t_{\mathrm{M}_{2}}\right]$ and the values of $I_{\mathrm{m}}$ in the range $\left[t_{\mathrm{m}_{1}}, t_{\mathrm{m}_{2}}\right]$ are stretched to the range $[0,255]$ with a linear scaling. So, the emphasised image $I_{\mathrm{E}}$ can be computed as:

$$
I_{\mathrm{E}}(i, j)=I_{\mathrm{m}, 2}(i, j)+\frac{I_{\mathrm{R}}(i, j)-I_{\mathrm{m}}(i, j)}{I_{\mathrm{M}}(i, j)-I_{\mathrm{m}}(i, j)}\left(I_{\mathrm{M}, 2}(i, j)-I_{\mathrm{m}, 2}(i, j)\right) .
$$

\subsection{Orientation Extraction}

Let $I$ be the initial image restricted to the significant mask obtained in the segmentation and with the ridge-valley structure emphasised as previously described.

The orientation extraction procedure is composed of three steps: orientation estimation, spatial period computation, orientation refinement.

\subsubsection{Orientation Estimation}

We use a directional gradient based approach, where the image convolution is performed by a Gaussian kernel in a given direction, and a Gaussian derivative in the orthogonal direction. Given $r \in \mathbb{R}_{+}$, we define the function $K_{r, 0}:[-r, r]^{2} \rightarrow \mathbb{R}:$

$$
K_{r, 0}(s, t)=d(s) \cdot e^{-\left|\frac{d(s)}{\sigma_{1}}\right|^{2 \alpha_{1}}} \cdot e^{-\left|\frac{d(t)}{\sigma_{2}}\right|^{2 \alpha_{2}}}
$$

whered $(t)=t-\left(\frac{r}{2}+1\right)$, and $\sigma_{1}, \alpha_{1}, \sigma_{2}$ and $\alpha_{2}$ are real positive parameters. We select $N_{\mathrm{A}}$ equally spaced angles $\theta_{1}, \ldots, \theta_{N_{\mathrm{A}}} \in[0, \pi)$ and define the following group of directional gradient kernels:

$$
K_{r, k}(i, j)=K_{r, 0}\left(i \cos \theta_{k}+j \sin \theta_{k},-i \sin \theta_{k}+j \cos \theta_{k}\right),
$$

where $k=1, \ldots, N_{\mathrm{A}}, i=1, \ldots, r_{1}, j=1, \ldots, r_{2}$. The response to $k$-th kernel gives the directional image derivatives along the direction with angle $\theta_{k}$.

An orientation estimation is extracted as follows: we convolve the image with each kernel $K_{r, k}$, compute the absolute value of the response, and smooth it with a Gaussian filter; we call $W_{k}$ the resulting matrix. Let $\phi_{k}=\left(\theta_{k}+\frac{\pi}{2}\right) \bmod \pi ; \mathcal{O}_{k}=W_{k} e^{2 i \phi_{k}}$ is a complex matrix with highmagnitude elements where ridges flow along the orientation with angle $\theta_{k}$. The desired orientation field $\mathcal{O}$ is computed as:

$$
\mathcal{O}(i, j)=\frac{\sum_{k=1}^{N_{\mathrm{A}}} W_{k}(i, j) \cdot e^{2 i \phi_{k}}}{\sum_{k=1}^{N_{\mathrm{A}}} W_{k}(i, j)}=\frac{\sum_{k=1}^{N_{\mathrm{A}}} \mathcal{O}_{k}(i, j)}{\sum_{k=1}^{N_{\mathrm{A}}} W_{k}(i, j)} .
$$

Notice that orientation angles are doubled in this process, thus giving a continuous field. A final Gaussian smoothing and an absolute value normalisation are performed, then the phase angles of $\mathcal{O}$ are halved.

\subsubsection{Spatial Period Computation}

From the orientation field $\mathcal{O}$ we can estimate the distance between two consecutive ridges. Consider the set of equally spaced gridded points on $\mathcal{O}$

$$
\left\{\left(i_{n}, j_{n}\right) \mid n=1, \ldots, N_{\mathrm{P}}\right\} .
$$

For each point $\left(i_{n}, j_{n}\right)$ consider the fixed-length segment centred at $\left(i_{n}, j_{n}\right)$, orthogonal to the orientation $\mathcal{O}\left(i_{n}, j_{n}\right)$, and pick $N_{\mathrm{S}}$ points on it. For each of these points an orientation $o_{n, k} \in \mathbb{C a n d}$ 
a value $v_{n, k} \in[0,255]$ with $k=1, \ldots, N_{\mathrm{S}}$ are obtained by respectively interpolating the field $\mathcal{O}$ and the image $I$.

In order to consider the $n$-th segment sufficiently reliable, a minimum absolute value threshold $\tilde{t}$ is chosen and the condition $\min \left\{\left|o_{n, k}\right|\right\}_{k=1, \ldots, N_{\mathrm{S}}}>\tilde{t}$ must be fulfilled, otherwise the segment is skipped. For each reliable segment, say the $n$-th one, consider the discrete signal

$$
v_{n}[k]=v_{n, k} k=1, \ldots, N_{\mathrm{S}},
$$

apply a lowpass filter, and compute the Fourier spectrum. The spatial frequency $f_{\mathrm{s}, n}$ along the $n$ th segment is the first peak after the zero-frequency one. The spatial frequency for the whole image can be computed as

$$
f_{\mathrm{s}}=\frac{1}{N_{\mathrm{P}}} \sum_{n=1}^{N_{\mathrm{P}}} f_{\mathrm{s}, n}
$$

The spatial period $T_{\mathrm{S}}$, i.e. the distance between two consecutive ridges, is given by $T_{\mathrm{S}}=\frac{N_{\mathrm{S}}}{f_{\mathrm{S}}}$.

\subsubsection{Orientation Refinement}

Let $F$ be a rectangular region in the image $I$, and $\mathcal{F}: F \rightarrow \mathbb{C}$ be an orientation field, defined in $F$, possibly given by the initial estimation $\mathcal{O}$; we denote with $\mathcal{F}(\mathbf{x})$ the orientation $\mathcal{F}(x, y)$ at point $\mathbf{x}=(x, y)^{\mathrm{T}} \in F$.

The refinement process is defined by two operators. Let $R \in \mathbb{N}$, we select $N_{\mathrm{C}}$ points $\mathcal{C}_{R}=$ $\left\{\mathbf{r}_{i}\right\}_{i=1, \ldots, N_{\mathrm{C}}} \subset \mathbb{R}^{2}$ from the circumference of radius $R$ centred at $\mathbf{0}$. We define the adjuster $\mathcal{G}_{\mathrm{A}}$ of the field $\mathcal{F}(\mathbf{x})$ as the following orientation field:

$$
\mathcal{G}_{\mathrm{A}}(\mathbf{x})=\frac{1}{N_{\mathrm{C}}} \sum_{k=1}^{N_{\mathrm{C}}} \operatorname{sgn}\left[a\left(\mathbf{x}, \mathbf{r}_{k}\right)\right] a\left(\mathbf{x}, \mathbf{r}_{k}\right)^{2} \mathcal{F}\left(\mathbf{x}+\mathbf{r}_{k}\right),
$$

Where

$$
a\left(\mathbf{x}, \mathbf{r}_{k}\right)=\mathfrak{R}\left\{\frac{\mathcal{F}\left(\mathbf{x}+\mathbf{r}_{k}\right)}{\left|\mathcal{F}\left(\mathbf{x}+\mathbf{r}_{k}\right)\right|} \frac{\left(\mathbf{r}_{k} \cdot 1-i \mathbf{r}_{k} \cdot \mathrm{\jmath}\right)}{\left\|\mathbf{r}_{k}\right\|}\right\},
$$

land jare the usual vectors of the canonical base for $\mathbb{R}^{2}, i$ is the imaginary unit, $\Re$ gives the real part of a complex number, -denotes the inner product, $\|\cdot\|$ is the Euclidean norm in $\mathbb{R}^{2}$ and $|\cdot|$ is the absolute value in $\mathbb{C}$. We call adjusted field the orientation field $\mathcal{A F}$ obtained as:

$$
\begin{aligned}
\mathcal{A F}_{0}(\mathbf{x})^{2} & =(1-s) \mathcal{F}(\mathbf{x})^{2}+s \mathcal{G}_{\mathrm{A}}(\mathbf{x})^{2}, \\
\mathcal{A F}(\mathbf{x}) & =\frac{\mathcal{A F}_{0}(\mathbf{x})}{\left|\mathcal{A F}_{0}(\mathbf{x})\right|} \max \left(|\mathcal{F}(\mathbf{x})|,\left|\mathcal{G}_{\mathrm{A}}(\mathbf{x})\right|\right),
\end{aligned}
$$

where $s \in(0,1)$ is a small parameter.

The smoother $\mathcal{G}_{\mathrm{S}}$ is the other operator and it is defined as follows:

$$
\mathcal{G}_{\mathrm{S}}(\mathbf{x})=\frac{1}{N_{\mathrm{C}}} \sum_{k=1}^{N_{\mathrm{C}}} \operatorname{sgn}\left[f_{d}\left(\mathbf{x}, \mathbf{r}_{k}\right)\right] a\left(\mathbf{x}, \mathbf{r}_{k}\right)^{2} \mathcal{F}\left(\mathbf{x}+\mathbf{r}_{k}\right),
$$

where $a\left(\mathbf{x}, \mathbf{r}_{k}\right)$ is defined as above and

$$
f_{d}\left(\mathbf{x}, \mathbf{r}_{k}\right)=\mathfrak{R}\left\{\mathcal{F}\left(\mathbf{x}+\mathbf{r}_{k}\right) \overline{\mathcal{F}(\mathbf{x})}\right\},
$$


where $\overline{\mathcal{F}(\mathbf{x})}$ is the complex conjugate of $\mathcal{F}(\mathbf{x})$. We call smoothed field the orientation field $\mathcal{S F}$ obtained as:

$$
\begin{aligned}
\mathcal{S F}_{0}(\mathbf{x})^{2} & =(1-s) \mathcal{F}(\mathbf{x})^{2}+s \mathcal{G}_{\mathrm{S}}(\mathbf{x})^{2}, \\
\mathcal{S F}(\mathbf{x}) & =\frac{\mathcal{S F}_{0}(\mathbf{x})}{\left|\mathcal{S F}_{0}(\mathbf{x})\right|} \max \left(|\mathcal{F}(\mathbf{x})|,\left|\mathcal{G}_{\mathrm{S}}(\mathbf{x})\right|\right) .
\end{aligned}
$$

The key operator of our procedure for orientation refinement is the smoother, that succeeds in reconstructing and giving global coherence to a noisy orientation field. The drawback of its application is the shifting effect it has on loops; since we iteratively apply the smoothing operator, we need a reliable mask where loops are in the background. The adjuster has the converse effect on loops, giving them back their initial position and enhancing their rounded shape.

The adjusting operator has a nice effect on loops, whereas strongly damages the deltas structure, hence it can be used to detect them. Let $\mathcal{O}_{\mathrm{S}}$ be the orientation estimated with directional gradient filters of sizer $=\frac{1}{2} T_{\mathrm{S}}$, and $\mathcal{O}_{\mathrm{B}}$ be the orientation estimated with directional gradient filters with sizer $=\frac{3}{2} T_{\mathrm{S}}$, where $T_{\mathrm{S}}$ is the spatial period. We compute a difference mask $D$ between the adjusted field $\mathcal{A O}_{\mathrm{S}}$ of $\mathcal{O}_{\mathrm{S}}$ and the adjusted field $\mathcal{A O}_{\mathrm{B}}$ of $\mathcal{O}_{\mathrm{B}}$. Since small variations of the orientation may also appear outside deltas, small connected components are filtered out; a final dilation is performed on the mask, to be sure to cover deltas.
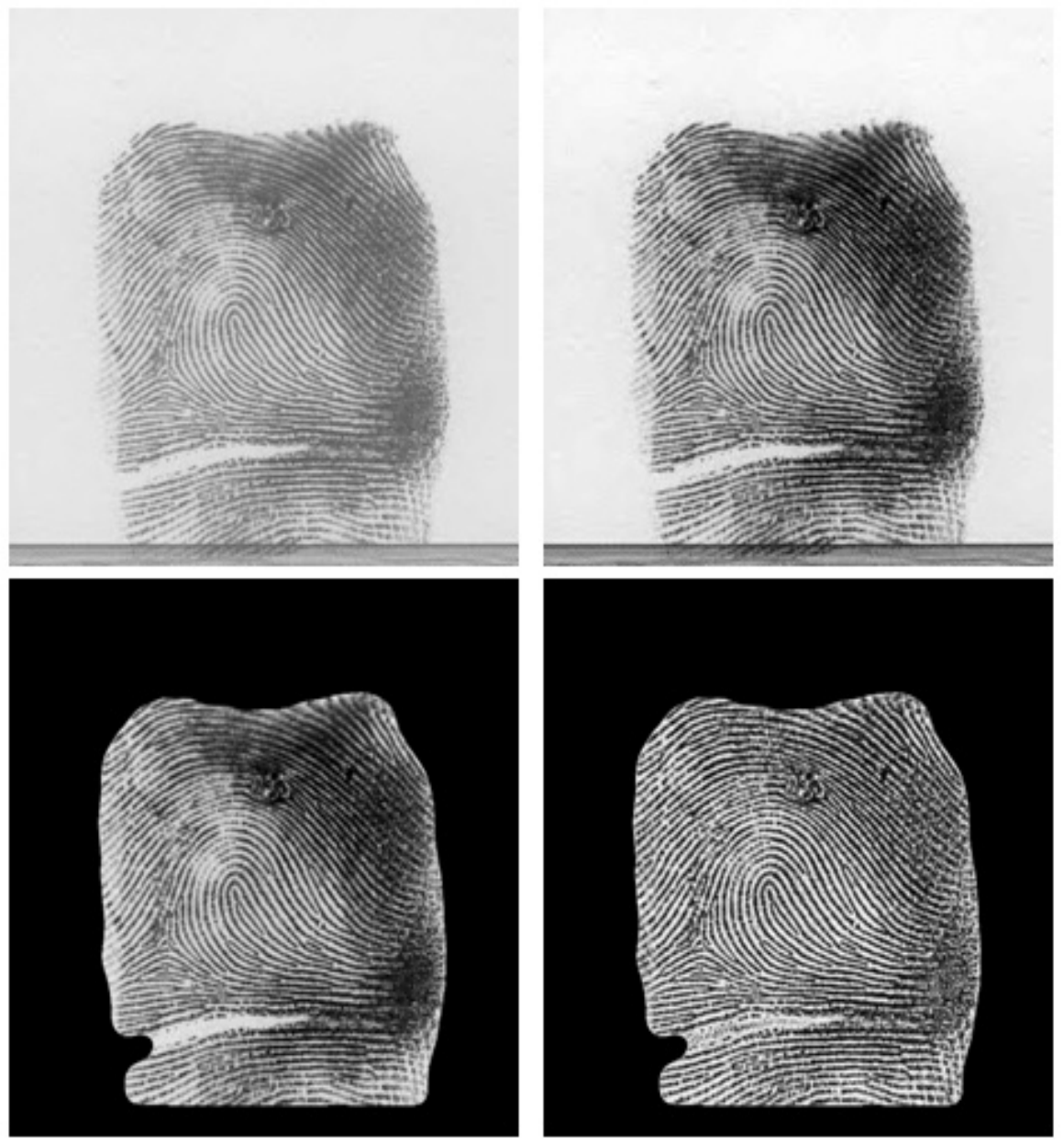

Figure 1. From top left: initial image, equalisation, segmentation and ridge amplification 

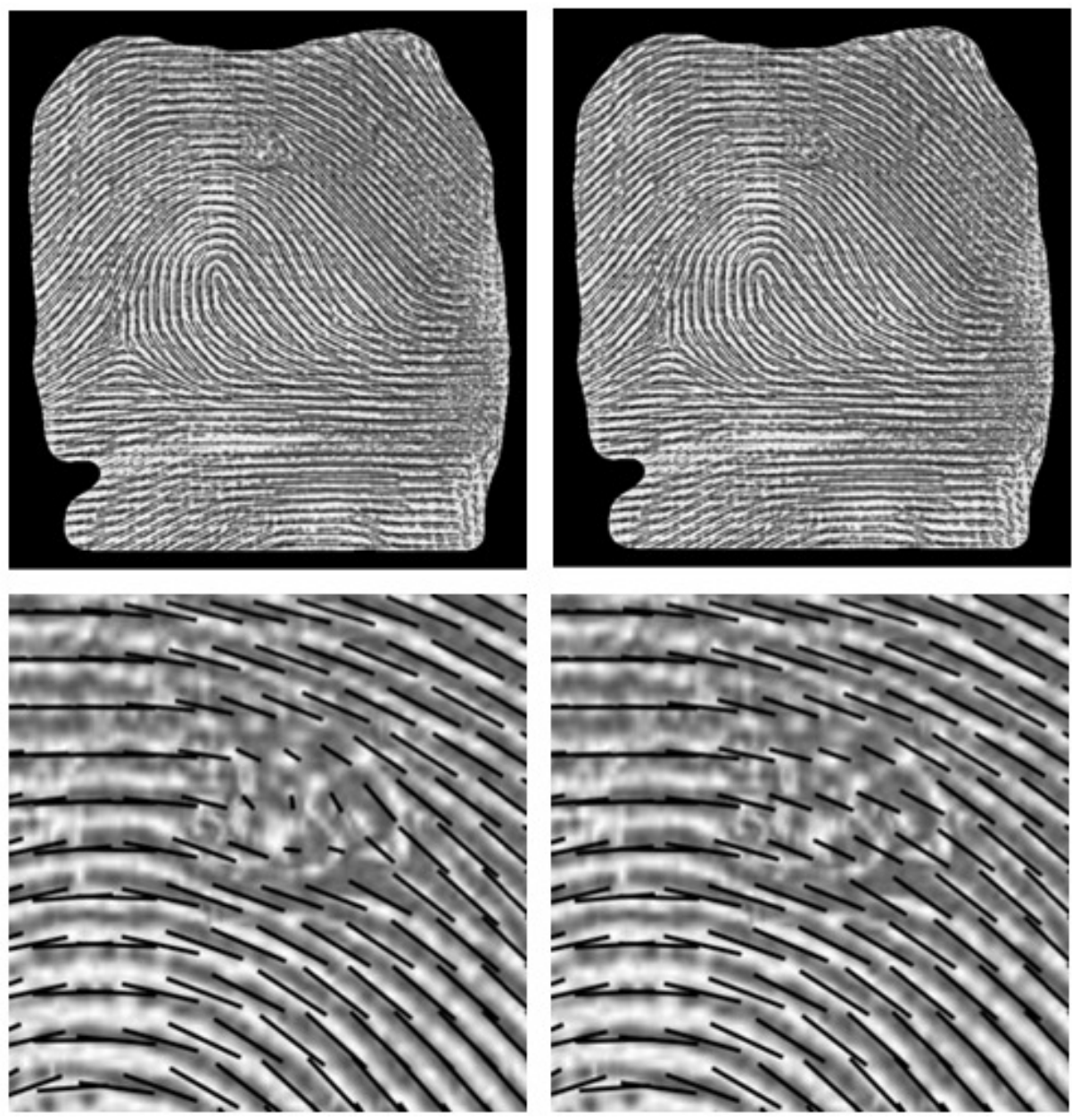

Figure 2. From top left: the extracted orientation field, the result of the refinement procedure, the magnification of a noisy area of the extracted orientation field, and the magnification of the same area after the refinement procedure

A mask $M_{\mathrm{CD}}$ with both cores and deltas is computed; $M_{\mathrm{CD}}$ is then eroded, so that it does not completely cover singularities. Small connected components, generated by noise and artifacts, are removed.

In our algorithm masks are matrices with values 0 or 255 ; in the following we use logical operators between masks, thus considering them with false elements in correspondence of 0 values, and with true elements in place of 255 values.

To get a loops-only mask $M_{\mathrm{L}}$, we need to join the information coming from $D$, with deltas, and from $M_{\mathrm{CD}}$, with cores too:

$$
M_{\mathrm{L}}(i, j)=M_{\mathrm{CD}}(i, j) \wedge \operatorname{not} D(i, j),
$$

where $\Lambda$ and "not" are the usual logical operators. Since during the creation of $M_{\mathrm{CD}}$ we eroded the mask, $M_{\mathrm{L}}$ may not entirely cover the loops: we need to perform a dilation. To finally get a noloops mask $M_{\mathrm{NL}}$ we do 


$$
M_{\mathrm{NL}}(i, j)=\operatorname{not} M_{\mathrm{L}}(i, j) .
$$

An iterative application of the smoothing operator within the mask $M_{\mathrm{NL}}$ can deeply improve the orientation estimation. Let us call $\mathcal{O}_{0}$ the orientation field obtained in the extraction procedure, and $M_{0}$ the starting mask $M_{\mathrm{NL}}$. At the step $k=1,2, \ldots$ the orientation field $\mathcal{O}_{k}$ is computed applying the smoothing operator. A difference mask $M_{k}$ between $\mathcal{O}_{k}$ and $\mathcal{O}_{k-1}$ is computed and intersected with the previous mask $M_{k-1}$, then a slight erosion is performed to guarantee convergence. The iterative procedure halts when no more points change orientation due to the smoothing operator, i.e. when $M_{k}$ is false everywhere.
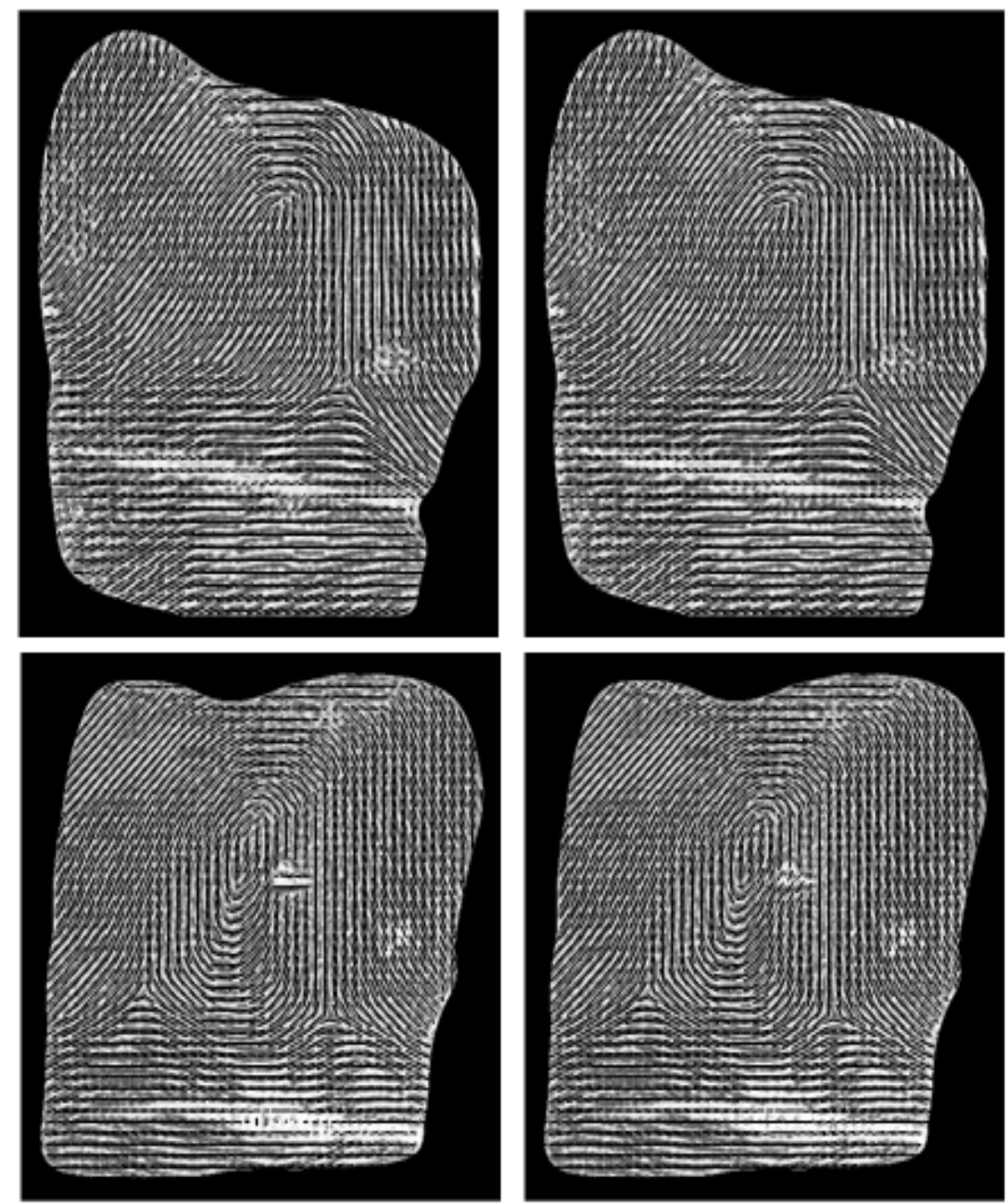

Figure 3. Two fingerprints from NIST Special Database 9 exhibits the orientation regularisation performance. In the first column the orientation extraction outcome is shown, while the second column presents the results of the orientation regularisation procedure.

Due to the shifting effect of the smoother on loops, the last step must be the application of the adjuster over them; in this way the initial position of loops is recovered and the drawbacks of the smoother are neutralised. 

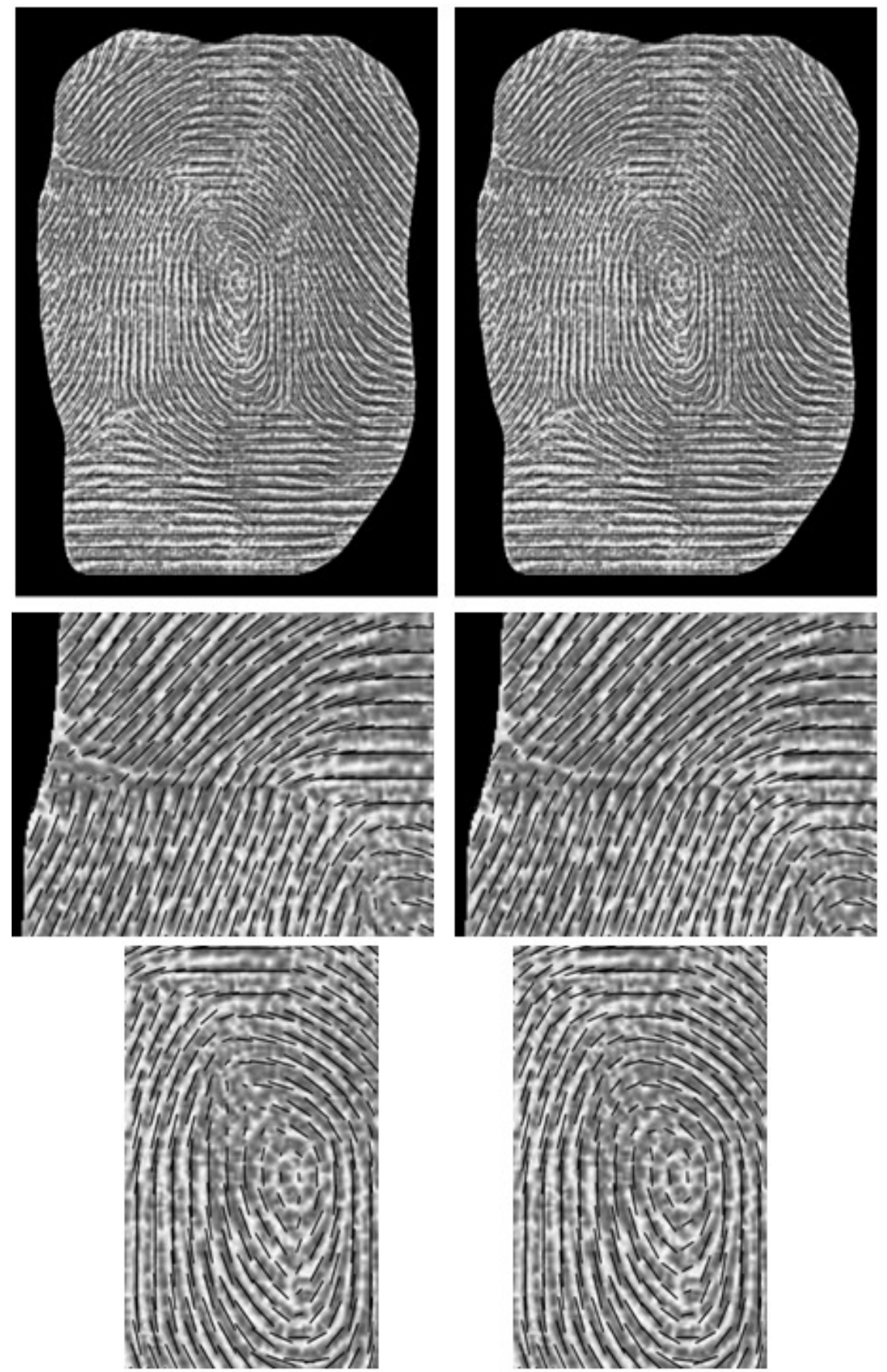

Figure 4. On the left the initial extracted orientation is presented, and on the right the related refined orientation. In the topmost line the whole image is shown, in the second and third lines two magnification of that image are exhibited for better understanding of our algorithm performance. 

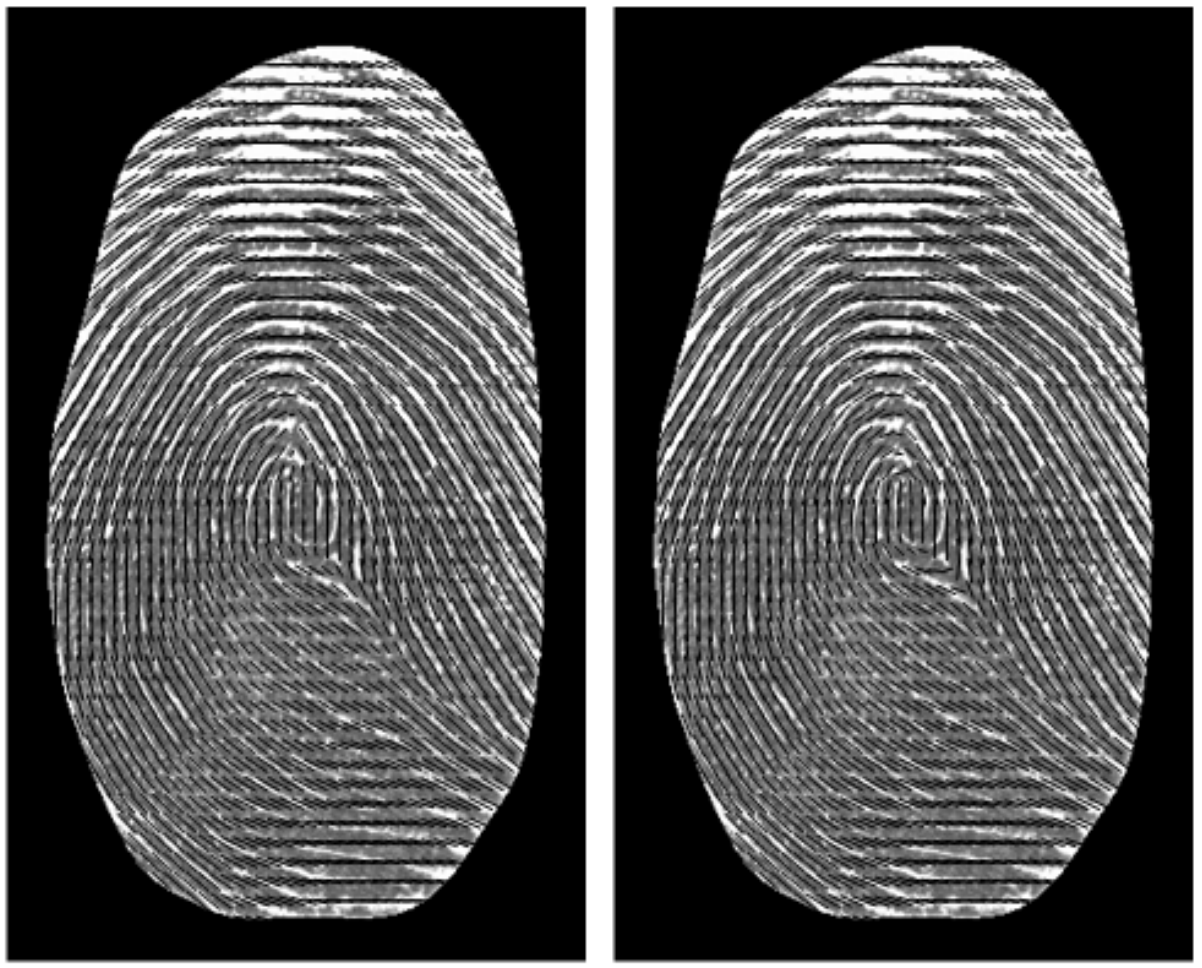

Figure 5. The effects of regularisation on another fingerprint image: on the left the orientation extraction, on the right the results of the orientation regularisation procedure.

\section{EXPERIMENTAL RESULTS}

We describe some of the results obtained in the numerical experience with the proposed method. In particular, we first show the outcome of the preliminary steps, such as image equalisation, segmentation, and ridge amplification; then we show the output of the orientation estimation and the orientation regularisation on five fingerprint images.

In Figure 1 we can see the effects of the preprocessing stage, i.e. image equalisation, segmentation, and ridge amplification. The parameters used to generate these images are: $t_{\mathrm{L}}=0.25, t_{\mathrm{R}}=0.5, \tau_{\mathrm{V}}=2.55, t_{\mathrm{M}_{1}}=0, t_{\mathrm{M}_{2}}=64, t_{\mathrm{m}_{1}}=192, t_{\mathrm{m}_{2}}=230$. The first image of Figure 1, starting from top left, is taken as an input to the algorithm described in Section 2; the second image is the result of the equalisation procedure, the third picture shows the segmentation, and in the last one the outcome of the ridge amplification step is exhibited. Notice the enhancement of ridge-valley structure, a more uniform brightness over the whole image, and the sharp detection of the significant part of the fingerprint image.

In Figure 2 we can see the initial orientation estimation in the top-left image, and the refined orientation in the top-right; due to the small image dimensions, in the bottom line of Figure 2, we exhibit also the magnification of a noisy area where our method performs very well. To compute the orientation estimation we used the parameters $r=15, \sigma_{1}=1, \alpha_{1}=2, \sigma_{2}=0.85, \alpha_{2}=2$, $N_{\mathrm{A}}=36, N_{\mathrm{S}}=31, \tilde{t}=0.25$. Figure 4 shows the refinement procedure on another fingerprint using the same parameters: in the top line of Figure 4 there are the initial extracted orientation field and result of the orientation refinement procedure on the whole image; in the second and 
third lines there are the magnifications of two meaningful areas, where the good behaviour of our method is clear.

The proposed algorithm performs very well in areas with a weak signal, such as creases, and in noisy parts of the image: the orientation there reconstructed exhibits high coherence with neighbouring areas. Figure 3provides a graphical explanation of such a good behaviour on a couple of fingerprint images.

The proposed method presents a good behaviour also around loops: if the loop structure has been weakened during the orientation extraction stage, our refinement procedure is able to recover it; this phenomenon is clarified by the results presented in Figure 5.

\section{CONCLUSION}

This paper presents a preprocessing procedure, along with a reliable algorithm to extract the orientation field and to improve it.

The proposed method still has to be tested against a database with ground truth information; this will help us tuning the parameters up and knowing their inner relationships; furthermore, testing our algorithm against such databases will let us compute the accuracy and computational time of the proposed method. Many databases can be found over the Internet, for instance the NIST Special Databases, which will be used in future studies to enhance the present algorithm and to extend it taking into account minutiae extraction.

\section{REFERENCES}

[1] Zhang, D. D. (2013). Automated biometrics: Technologies and systems (Vol. 7). Springer Science \& Business Media.

[2] Biradar, V. G., \& Sarojadevi, H. (2014). Fingerprint Ridge Orientation Extraction: A Review of State of the Art Techniques. International Journal of Computer Applications, 91(3).

[3] Maltoni, D., Maio, D., Jain, A., \& Prabhakar, S. (2009). Handbook of fingerprint recognition. Springer Science \& Business Media.

[4] Guo, J. M., Liu, Y. F., Chang, J. Y., \& Lee, J. D. (2014). Fingerprint classification based on decision tree from singular points and orientation field. Expert Systems with Applications, 41(2), 752-764.

[5] Liu, Q., Peng, K., Liu, W., Xie, Q., Li, Z. Y., Lan, H., \& Jin, Y. (2014). Fingerprint singular points extraction based on orientation tensor field and Laurent series. Journal of Central South University, 21, 1927-1934.

[6] Ellingsgaard, J., Sousedik, C., \& Busch, C. (2014, March). Detecting fingerprint alterations by orientation field and minutiae orientation analysis. In Biometrics and Forensics (IWBF), 2014 International Workshop on (pp. 1-6). IEEE.

[7] Krish, R. P., Fierrez, J., Ramos, D., Ortega-Garcia, J., \& Bigun, J. (2015). Pre-registration of latent fingerprints based on orientation field. IET Biometrics, 4(2), 42-52.

[8] Qi, J., Yang, S., \& Wang, Y. (2005). Fingerprint matching combining the global orientation field with minutia. Pattern Recognition Letters, 26(15), 2424-2430.

[9] Tico, M., \& Kuosmanen, P. (2003). Fingerprint matching using an orientation-based minutia descriptor. IEEE Transactions on Pattern Analysis and Machine Intelligence, 25(8), 1009-1014. 
[10] Kulkarni, J. V., Patil, B. D., \& Holambe, R. S. (2006). Orientation feature for fingerprint matching. Pattern Recognition, 39(8), 1551-1554.

[11] Jiang, X., Liu, M., \& Kot, A. C. (2004, August). Reference point detection for fingerprint recognition. In Pattern Recognition, 2004. ICPR 2004. Proceedings of the 17th International Conference on (Vol. 1, pp. 540-543). IEEE.

[12] Zhu, E., Yin, J., Hu, C., \& Zhang, G. (2006). A systematic method for fingerprint ridge orientation estimation and image segmentation. Pattern Recognition, 39(8), 1452-1472.

[13] Oliveira, M. A., \& Leite, N. J. (2008). A multiscale directional operator and morphological tools for reconnecting broken ridges in fingerprint images. Pattern Recognition, 41(1), 367-377.

[14] Chen, X., Tian, J., Zhang, Y., \& Yang, X. (2006, January). Enhancement of low quality fingerprints based on anisotropic filtering. In International Conference on Biometrics (pp. 302-308). Springer Berlin Heidelberg.

[15] Lee, K. C., \& Prabhakar, S. (2008, September). Probabilistic orientation field estimation for fingerprint enhancement and verification. In Biometrics Symposium, 2008. BSYM'08 (pp. 41-46). IEEE.

[16] Turroni, F., Maltoni, D., Cappelli, R., \& Maio, D. (2011). Improving fingerprint orientation extraction. IEEE Transactions on Information Forensics and Security, 6(3), 1002-1013.

[17] Sherlock, B. G., \& Monro, D. M. (1993). A model for interpreting fingerprint topology. Pattern recognition, 26(7), 1047-1055.

\section{AUTHORS}

Pierluigi Maponi is associate professor in Numerical Analysis at the School of Science and Technology of Camerino University. Besides fingerprint analysis, his interest for image processing also concerns automatic age estimation, biomedical imaging and diagnostics, satellite imaging. Other research fields are numerical linear algebra, inverse problems and applications, computational fluid dynamics, hazard evaluation for water-related events.

Riccardo Piergallini is full professor in Geometry at the School of Science and Technology of Camerino University. The main field of his scientific activity is lowdimensional topology. In particular, he is interested in the theory of branched coverings, as a tool for representing manifolds and studying various topological and geometric structures on them. Recently, he started to consider computational applications of topology and geometry, specially the ones concerning spatial modeling, image processing, computer graphics and artificial vision.

Filippo Santarelli is a $\mathrm{PhD}$ student at the School of Science and Technology of Camerino University. His research interests are fingerprint analysis and voice recognition.
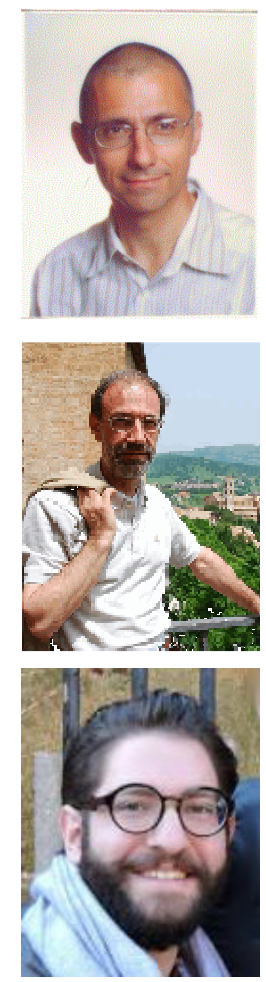